\title{
SÉANCE DU 28 JUIN 1918
}

Présidenge de M. F. Camus, Archiviste.

Après lecture du procès-verbal dont la rédaction est adoptée, M. Giraudias donne quelques détails sur la Note ci-après :

\section{Notes de botanique systématique. VI}

\author{
PAR M. L. GIRAUDIAS.
}

Viola scotophylla Jord.

Cette plante ne figure pas dans le Catalogue des plantes $d u$ Loiret, publié par M. Julien Crosnier, non plus que dans les deux suppléments.

Elle n'est cependant pas très rare aux environs d'Orléans. J'en possède un exemplaire, sans feuilles automnales à la vérité, mais cependant très reconnaissable, récolté par moi au printemps de 1901, dans les haies qui bordent le chemin de halage, commune de la Chapelle Saint-Mesmin. Je l'ai vue également dans les anciennes tranchées remontant, parait-il, à l'occupation anglaise, qui se trouvent au bois de l'Ermitage.

\section{Quelques Viola de la Charente-Inférteure.}

J'ai retrouvé récemment un petit fascicule contenant plusieurs parts d'espèces du genre Viola récoltées par moi en 1882 dans la Charente-Inférieure et que j'avais négligé d'étudier. En m'aidant des tableaux dichotomiques contenus dans la Flore de M. Rouy, je suis parvenu à leur donner des noms que je considère comme exacts.

Ce sont : dans le groupe du $V$. tricolor :

Viola Kitaibeliana R. s. var. parviflora Reichb. - Aulnay. (Plante grêle, dressée, presque constamment uniflore.) 
Viola Deseglisei Jord. Aulnay, à Palud.

V. mentita Jord. Villemorin, dans un champ de lin à Presles.

v. lepida Jord. Aulnay. Les fleurs assez grandes à pétales supérieurs d'un beau violet la distinguent facilement des autres variétés du même groupe.

Dans le groupe du $V$. hirta:

V. hirta var. fraterna Reichb. - Aulnay. Mes échantillons répondent à la description de M. Rouy, mais ils offrent cette particularité que les pétales supérieurs sont dépourvus de poils.

J'ai noté également quelques échantillons de cette espèce dont l'éperon serait mucroné. Je n'ai pu revoir sur la plante sèche ce caractère que j'avais observé sur la plante vivante. Un seul éperon est visible, il est longuement atténué en pointe et recourbé ascendant. Ce serait, sauf le léger détail rappelé plus haut, le $V$. revoluta Heuf. d'après M. Rouy.

M. Joly, instituteur honoraire à Orléans, botaniste zélé et méticuleux, m'a fait récolter dans la forêt d'Orléans plusieurs pieds de $V$. Riviniana dont l'éperon portait un petit éperon superposé.

Cas d'albinisme dans le gente Geranium.

Geranium columbinum. - Seine: Paris, Vaugirard, juin 1896. - Leg. E. Bécourt.

G. dissectum. - Gard : le Vigan, 26 mai 1877. Leg. L. Anthouard.

G. modestum Jord. - Aveyron : Conques, 11 mai 1875; Leg. G. Chastaingt.

G. molle. - Charente-Inférieure : Châtelaillon, 10 mai 1883 , ispe legi.

G. phæum. - Allier : Saint-Pourçain, juin 1884. Leg. C. Bourgougnon. Hausser.

G. pyrenaicum. - Haut-Rhin : Markich, 19 juin 1891. Leg. G. Robertianum. - Ariège : Foix, כ juin 1889, ipse legi.

Lavatera arborea $\mathbf{L}$.

J'ai récolté cet arbuste en juillet 1916 à Pontaillac, près 
Royan, dans un jardin abandonné. J'ignore si cette Malvacée y avait été cultivée précédemment. Le $L$. arborea se trouve fréquemment dans l'Ouest à l'état sporadique. Lloyd, dans la Flore de l'Ouest, ne le signale pas dans la Charente-Inférieure; mais la Société Rochelaise l'a distribué de Saint-Georges d'Oléron, sous le $\mathrm{n}^{\circ} 2820$, recueilli par M. Réau.

Carduus tenuiflorus Curt.

Cette espèce n'est pas indiquée dans la péninsule scandinave. Cependant j'en ai reçu un bel exemplaire dans un fascicule de plantes suédoises. La plante désignée à tort sous le nom de C. acanthoides a été récoltée le 2 juillet 1893 par M. J. A. Holm. L'étiquette porte l'indication géographique suivante : Medelpad Wifstavay (Carlau). Il est à remarquer que cette plante vient surtont dans les décombres et qu'il est admissible qu'elle ait des tendances à se répandre.

Sonchus arvensis var. riparius Magn.

A Royan, dans le bois de Vallières, sur les bords du ruisseau, j'ai récolté un Sonchus de très grande taille que je ne puis rapporter qu'à cette variété. Elle est très assimilable aux échantillons distribués par M. Magnier dans son $\mathrm{Fl}$. selecta, sous le $\mathrm{n}^{\circ} 1224$, numéro indiqué comme référence par M. Rouy, (Fl. de Fr., IX, p. 205). Elle a toutefois des calathides moins nombreuses et les feuilles moins longues (de 20 à $25 \mathrm{~cm}$. au plus). Les fleurs sont très grandes, abondamment pourvues de poils glanduleux.

Il est à noter que cette variété n'a pas échappé à l'œil perspicace de Lloyd qui en dit (loc. cit., éd. 4, p. 241) : "Quelquefois haut de 10-15 décimètres, sur le bord des eaux; en cet état, il ne faut pas le confondre avec le suivant (S. palustris) ').

Peu d'espèces végétales peuvent mieux que le $S$. arvensis revendiquer la qualification de a variable ». Mot vague en vérité et qui, je le crains, ne correspond pas à une réalité bien définie. Je possède dans mon herbier le $S$. arvensis d'assez nombreuses localités. Il ne s'y trouve pas deux parts 'qu'on pourrait réunir sous un même vocable, comme étant exactement synonymes. Ce qui indique que ce type linnéen renferme un très grand nombre de formes, ou mème de races qu'on 
pourrait distinguer, qui se reproduisent peut-être avec leurs caractères propres et dont l'École jordannienne, en les cultivant, aurait fait autant d'espèces.

Jasione maritima Duf.

M. Rouy, dans sa Flore de France, X, p. 92, rapporte cette plante comme race au $J$. montana dont la caractéristique est de ne pas porter de rosettes stériles. Or, dans une excursion que je fis en juillet 1916 entre Saint-Palais-sur-Mer et la Grande Côte, près Royan (Charente-Inférieure), sur des falaises sablonneuses avoisinant le Puits de Loture, j’ai récolté des échantillons du J. maritima manifestement pourvus d'assez nombreuses rosettes. Il n'y a aucun doute à émettre sur l'identification de la plante. Or Willkomm et Lange dans leur Prodromus floræ hispanicæ avaient fait la même remarque que moi et rapporté le J. maritima qui croît aussi sur les côtes océaniennes de l'Espagne comme variété au J. humilis.

Je ne crois pas avoir à discuter ce rapprochement entre une plante montagnarde et une plante maritime, mais toujours estil que la plante de nos côtes de l'Ouest n'appartient pas au groupe du $J$. montana et doit être classée dans une espèce différente. Elle ne doit pas être confondue avec la var. littoralis Fr., plante septentrionale signalée en Normandie et en Bretagne.

J'ai examiné les Jasione du Muséum sans y trouver d'échantillons caractéristiques pouvant être assimilés avec certitude à la plante charentaise.

J'ai été plus heureux avec l'herbier Cosson, que son très obligeant conservateur, M. Jeanpert, a mis à ma disposition avec une complaisance dont je ne saurais trop le remercier.

J'ai trouvé dans cette collection deux exemplaires portant des rosettes : l'un récolté par A. Guillon à Royan en juin 1842; l'autre rapporté de la Teste (Gironde), le 14 juillet 1847, par Cosson lui-même.

Dans cette dernière part les rosettes commencent à se développer.

Dans ses exsiccata, M. Magnier a publié, sous le $\mathrm{n}^{\circ} 2519$, un J. Maritima que M. Rouy n'indique pas comme référence, mais dont les exemplaires, bien que ne portant pas de rosettes, ne laissent subsister aucun doute sur leur identité avec la plante 
L. GiRaudias. - NOTES de Botanique systématique.

de Royan. Ces exemplaires ont été récoltés à Arès (Gironde) par H. Brochon.

Je signalerai encore dans l'herbier Cosson un $J$. montana récolté à Saint-Aigulin (Charente-Inférieure) par A. G. de Bévès (?), lequel a des analogies avec la plante de Saint-Palais, sans cependant pouvoir lui être identifié. Sa singularité n'a pas échappé au collecteur qui a marqué sur son étiquette : aspect singulier.

Le $J$. maritima a des affinités avec le $J$. lusitanica DC., du Portugal.

Origanum creticum L.; O. vulgare L. var. macrostachyum Brot (R. X, p. 347).

On sait que cette « variété » présente cette singularité d'avoir des épis allongés prismatiques. J'ai rencontré assez souvent l'O. creticum mélangé au type, pour ainsi dire à l'état sporadique.

On trouve sur le même pied et dans la même inflorescence des épis normaux et des épis allongés. Exemple : les échantillons, d'ailleurs peu caractérisés, distribués par la Société Dauphinoise sous le $\mathrm{n}^{\circ} \mathbf{5} \mathbf{4 6 4}$.

Je constate semblable déformation dans d'autres espèces :

O. lirium Held. Eubée M. Dirphys, Heldreich (H. gr. norm., $\mathrm{n}^{\circ}$ 783/2).

O. humile Poir. Ain : Trévoux B., $\mathbf{n}^{\circ} 4061$.

O. hirtum Lk. Kerata (Constantine), juillet 1896, Leg. Reverchon, $\mathrm{n}^{\circ} 77$.

Je me demande s'il ne faut pas interpréter cette prétendue variété comme une déformation traumatique due à la piqûre d'un insecte.

Et cette supposition s'appuie sur des échantillons de l'Achillea Ageratum récoltés par Reverchon dans la Sierra de Cartama le 13 juillet 1888 et distribués sous le $n^{\circ} 295$, lequel présente le même aspect spiciforme que l’O. creticum.

Limodorum occidentale Rouy.

M. Rouy ne donne comme station de cette sous-espèce que les environs de Dœuil (Charente-Inférieure). Je l'ai rencontrée le 24 avril 1869 à Chaillot, commune de Fontcouverte, dans

T. LXV.

(SÉANCES) 9 
le même département. Cette précocité est remarquable. L'échantillon que j'ai conservé répond entièrement à la description de la Flore de France, et j'estime que M. Rouy a eu raison de séparer cette plante du type dont elle se distingue au premier coup d'œil. 


\section{$2 \mathrm{BHL}$ Biodiversity Heritage Library}

Giraudias, Louis. 1918. "Notes de botanique systématique. VI." Bulletin de la Société botanique de France 65, 125-130.

https://doi.org/10.1080/00378941.1918.10836063.

View This Item Online: https://www.biodiversitylibrary.org/item/8685

DOI: https://doi.org/10.1080/00378941.1918.10836063

Permalink: https://www.biodiversitylibrary.org/partpdf/159708

\section{Holding Institution}

Missouri Botanical Garden, Peter H. Raven Library

\section{Sponsored by}

Missouri Botanical Garden

\section{Copyright \& Reuse}

Copyright Status: Public domain. The BHL considers that this work is no longer under copyright protection.

This document was created from content at the Biodiversity Heritage Library, the world's largest open access digital library for biodiversity literature and archives. Visit BHL at https://www.biodiversitylibrary.org. 